\title{
Risk Factor Detection as a Metric of STARHS Performance for HIV Incidence Surveillance Among Female Sex Workers in Kigali, Rwanda
}

\author{
Sarah L. Braunstein ${ }^{*}, 1$, , Janneke H. van de Wijgert ${ }^{2,3}$, Joseph Vyankandondera ${ }^{2,4}$, \\ Evelyne Kestelyn ${ }^{2}$, Justin Ntirushwa ${ }^{2}$ and Denis Nash ${ }^{1,5}$ \\ ${ }^{I}$ Mailman School of Public Health, Columbia University, New York, USA \\ ${ }^{2}$ Projet Ubuzima, Kigali, Rwanda \\ ${ }^{3}$ Academic Medical Center of the University of Amsterdam, Department of Internal Medicine, and Amsterdam Institute \\ for Global Health and Development, Amsterdam, The Netherlands \\ ${ }^{4}$ Belgian Development Agency, Kigali, Rwanda \\ ${ }^{5}$ CUNY School of Public Health, New York, USA
}

\begin{abstract}
Background: The epidemiologic utility of STARHS hinges not only on producing accurate estimates of HIV incidence, but also on identifying risk factors for recent HIV infection.

Methods: As part of an HIV seroincidence study, 800 Rwandan female sex workers (FSW) were HIV tested, with those testing positive further tested by BED-CEIA (BED) and AxSYM Avidity Index (Ax-AI) assays. A sample of HIVnegative (N=397) FSW were followed prospectively for HIV seroconversion. We compared estimates of risk factors for: 1) prevalent HIV infection; 2) recently acquired HIV infection (RI) based on three different STARHS classifications (BED alone, Ax-AI alone, BED/Ax-AI combined); and 3) prospectively observed seroconversion.

Results: There was mixed agreement in risk factors between methods. HSV-2 coinfection and recent STI treatment were associated with both prevalent HIV infection and all three measures of recent infection. A number of risk factors were associated only with prevalent infection, including widowhood, history of forced sex, regular alcohol consumption, prior imprisonment, and current breastfeeding. Number of sex partners in the last 3 months was associated with recent infection based on BED/Ax-AI combined, but not other STARHS-based recent infection outcomes or prevalent infection. Risk factor estimates for prospectively observed seroconversion differed in magnitude and direction from those for recent infection via STARHS.

Conclusions: Differences in risk factor estimates by each method could reflect true differences in risk factors between the prevalent, recently, or newly infected populations, the effect of study interventions (among those followed prospectively), or assay misclassification. Similar investigations in other populations/settings are needed to further establish the epidemiologic utility of STARHS for identifying risk factors, in addition to incidence rate estimation.
\end{abstract}

Keywords: HIV/AIDS, incidence, cross-sectional surveys, prospective studies, risk factors, Rwanda.

\section{INTRODUCTION}

Reliable information on HIV incidence is critical to public health practitioners and policymakers working in HIV prevention in order to identify target populations, evaluate the impact of HIV prevention interventions, and identify important trends in dynamic epidemics [1]. Data on risk factors for incident HIV infection help identify at-risk subgroups in immediate need of primary prevention interventions [2,3]. Despite these important applications, data on HIV incidence rates and risk factors are scant,

*Address correspondence to this author at the New York City Department of Health and Mental Hygiene, New York, USA; Tel: 347-396-7760; Fax: 347-396-7793; E-mail: braunstein.sarah@gmail.com

${ }^{\#}$ Current Affiliation: New York City Department of Health and Mental Hygiene, New York, USA. especially in sub-Saharan Africa where the majority of new HIV infections are occurring [4, 5], and where most information on risk factors is derived from HIV prevalence data, such as from the Demographic and Health Surveys (DHS) [6]. Key public health agencies, including the World Health Organization and US Centers for Disease Control and Prevention, have begun recommending strengthening of HIV incidence surveillance systems at the country and regional levels [7-11].

The Serologic Testing Algorithm for Recent HIV Seroconversion (STARHS) is a laboratory test-based approach that offers a promising alternative to prospective incidence measurement for incidence surveillance. Laboratory assays used under STARHS, such as the BEDCEIA (BED) [12] and AxSYM Avidity Index method (AxAI) [13], exploit immunologic properties of early HIV infection, such as development of HIV antibodies, to distinguish recent infections (RI) from long-term infections 
(LTI) in HIV-positive persons, enabling estimation of incidence in a cross-sectional sample of HIV-positive and negative individuals [9, 10, 14-24]. Further, if demographic and behavioral data are collected alongside biologic samples for STARHS testing, risk factors for RI, as distinguished from prevalent infection, can be identified.

The potential public health impact of the STARHS method lies in its epidemiologic utility, which we define as the ability of an assay or algorithm to: 1) accurately estimate HIV incidence rates, and 2) distinguish risk factors for RI from LTI when such differences exist, within a population sample of individuals tested for HIV and interviewed for behavioral and other risk factor information. The latter is particularly useful when factors associated with HIV transmission and acquisition shift over time, such that groups currently at risk for infection (eventual "incident cases") have different behavioral, demographic or other characteristics from those with LTI $[25,26]$. To date, few studies have evaluated STARHS from the perspective of epidemiologic utility. Instead, studies have focused on assessing assay validity, concluding that STARHS assays tend to misclassify certain individuals with LTI as RI [12, 17, 27-36]. At the population level, this often results in an overestimation of HIV incidence rates relative to prospectively observed seroconversion rates [18]. However, from an epidemiologic standpoint, a certain degree of assay/outcome misclassification may be less problematic. For example, an assay or algorithm that misclassifies certain individuals and overestimates HIV incidence can still be considered epidemiologically useful if associations and inference related to risk factors are not appreciably altered.

We conducted a combined cross-sectional survey (with STARHS testing) and prospective cohort study of female sex workers (FSW) at Projet Ubuzima in Kigali, Rwanda. An indepth analysis of the validity of STARHS-based incidence estimates in this population was reported separately, and found that HIV incidence rate estimates derived via the BED assay alone and BED combined with Ax-AI were similar to those based on prospective observation of HIV seroconversion [24]. However, incidence rate estimates derived via Ax-AI alone were substantially higher than those based on observed HIV seroconversion. The present analysis focused on risk factor estimation using STARHS plus behavioral interview data. Risk factor estimates (adjusted odds ratios) were compared for: 1) prevalent HIV infection; 2) RI based on three different STARHS RI classifications (BED alone, Ax-AI alone, BED/Ax-AI combined) with CD4 correction $(<200$ cells $/ \mu 1$ excluded from $\mathrm{RI})$; and 3 ) observed HIV seroconversion in the prospective study.

\section{MATERIALS AND METHODOLOGY}

\section{Study Design and Procedures}

The study was conducted by Projet Ubuzima, a nongovernmental medical research organization in Kigali, Rwanda. Between October 2006 and August 2007, 800 women participated in the cross-sectional survey with HIV, HSV-2 and pregnancy testing. Individuals testing HIV positive were further tested with the BED and Ax-AI assays, and CD4 cytometry. All women underwent a face-to-face interview for demographic and behavioral risk factor information. Women were eligible for the cross-sectional study if they were: $\geq 18$ years; at high risk for sexual exposure to HIV, defined as having exchanged sex for money at least once in the last month and/or currently having sex with multiple partners plus having sex at least twice per week (all enrolled women self-reported sex work); HIV serostatus unknown or last test negative; and willing and able to provide written informed consent.

Of 608 women identified as HIV-uninfected during the cross-sectional survey, 397 (65\%) who consented and were not pregnant were enrolled consecutively into a prospective cohort study. Cohort participants returned for five visits over two years for HIV counseling and testing (including condom provision), pregnancy and HSV-2 testing, and face-to-face interviewing. Twelve-month retention was $96 \%$, and median follow-up was 689 days (range: 0-836). Specimens from women who became HIV infected during follow-up were tested by CD4 cytometry. Women who tested positive for HIV, HSV-2 or pregnancy were referred for care and treatment. All HIV-positive study participants were ARTnaïve.

The study was approved by the National Ethics Committee and National HIV/AIDS Committee (CNLS) in Rwanda, and by Columbia University Medical Center's Institutional Review Board in the United States.

\section{Laboratory Assessments}

Blood specimens were tested for HIV by First Response (Premier Medical Corporation, India) and Uni-Gold (Trinity Biotech Plc, Ireland) rapid tests, with Capillus HIV-1/HIV-2 Rapid Test (Trinity Biotech Plc, Ireland) as a tie-breaker. HIV rapid test-positive results were confirmed by Murex HIV Ag/Ab Combination ELISA (Abbott Laboratories, Germany), and then tested further by CD4 cytometry at Rwanda's national reference laboratory in Kigali. HSV-2 infection was assessed by HerpeSelect 2 ELISA (Focus Technologies, USA) and pregnancy by the Fortress hCG test (Fortress Diagnostics, UK). HSV-2, pregnancy, HIV rapid and ELISA testing was done onsite at Projet Ubuzima.

Blood specimens from HIV-positive survey participants were tested by the BED and Ax-AI assays. BED testing was performed onsite following standard procedures as described in the literature $[23,24]$ and manufacturer's package insert (Calypte $^{\circledR}$ Biomedical Corporation, Oregon, USA), using a cutoff of OD-n $\leq 0.8$ to indicate RI. Ax-AI testing was performed by the Pediatric HIV Research Unit in South Africa using an unmodified AxSYM HIV-1/2gO ELISA (Abbott, USA), and following procedures described in the literature [13], with an avidity index $\leq 0.85$ indicating RI (Ax-AI cutoff based on personal communication with $B$. Suligoi).

\section{STATISTICAL METHODS}

\section{Outcome Definitions}

In the cross-sectional sample, a prevalent HIV case was a participant who tested positive on HIV rapid tests (with ELISA confirmation), irrespective of STARHS results. Prevalent cases represent undiagnosed HIV infections only in the sampled population, given the eligibility criterion of having an unknown HIV serostatus or a negative last HIV test. In analyses of BED and Ax-AI separately, RI cases 
were HIV-positive participants who were classified as RI by the BED or Ax-AI, respectively. In analyses using BED and Ax-AI combined, we counted as RI cases only those survey participants who tested HIV positive and were then classified as RI by both BED and Ax-AI assays. Additionally, individuals with $\mathrm{CD} 4<200$ cells $/ \mu 1$ were considered probable LTI cases regardless of STARHS results, and so individuals classified as RI but with $\mathrm{CD} 4<200$ cells/ $\mu 1$ were removed from analyses of recent infection [37], but examined in sensitivity analyses. In the prospective sample, HIV seroconversion date was estimated as the midpoint between the last negative and first positive HIV test $( \pm 3$-month interval).

\section{Analysis}

For the cross-sectional sample, odds ratios (OR) and $95 \%$ confidence intervals (CI) for prevalent HIV (based on HIV rapid testing), and for RI (based on STARHS classifications) were derived from logistic regression models, with HIVnegative participants as the comparison group for each. All OR are age-adjusted, and factors with $P<0.05$ were considered statistically significant.

For the prospective sample, Cox proportional hazards regression was used to estimate hazard ratios (HR) and 95\% CI for HIV seroconversion. Only results for baseline covariates are presented to enable comparison with logistic models for the cross-sectional sample. All HR are ageadjusted, and factors with $P<0.05$ were considered statistically significant.

We qualitatively compared the direction, magnitude, and statistical significance of putative risk factors for: 1) prevalent HIV; 2) RI based on three different STARHS classifications (BED alone, Ax-AI alone, and BED/Ax-AI combined); and 3) prospectively observed seroconversion in the cohort study. All statistical tests are two-sided. Data were analyzed using SAS, version 9.2 (SAS Institute, Inc., Cary, NC).

\section{RESULTS}

\section{Sociodemographics}

HIV-positive survey participants ( $\mathrm{N}=192)$, HIV-negative, non-enrolled survey participants $(\mathrm{N}=211)$, and prospective cohort participants $(\mathrm{N}=397)$ were similar on most sociodemographic characteristics (Table 1). Median age in the three groups was 27 (IQR: 23-32), 25 (23-31), and 24 (22-28), respectively. Approximately one-fifth of participants in all groups $(23 \%, 17 \%$, and $22 \%)$ had no formal schooling. Most participants $(93 \%, 91 \%$, and $91 \%)$ reported currently using at least one contraceptive method, with about three-quarters $(72 \%, 72 \%$, and $75 \%)$ reporting condom use by their last sex partner. The majority of women in the three groups had 1-2 pregnancies during their lifetimes. The proportion currently breastfeeding varied across the groups $(21 \%, 45 \%$ and $55 \%)$.

\section{Prevalent, Recent, and Incident HIV Infections}

In the cross-sectional survey, 192 women tested HIV positive for a prevalence of $24.0 \%(95 \% \mathrm{CI}, 21.0-27.0)$. Among 190 prevalent HIV cases with BED/Ax-AI results, $36(19 \%)$ were classified as RI by BED, and $56(30 \%)$ as RI by Ax-AI. Twenty-three participants $(12 \%)$ were classified as RI by both assays; $121(64 \%)$ as LTI by both assays; and $46(24 \%)$ were classified discordantly on the assays (i.e., recent on BED and long-term on Ax-AI, or vice versa). Two individuals concordant for RI on the assays (9\%) had CD4 $<200$ cells $/ \mu 1$, indicating possible LTI. Nineteen of the 397 cohort participants $(5 \%)$ seroconverted for HIV during 686.5 person-years of follow-up (2.8 per 100 person-years).

\section{Risk Factors for Prevalent and Recent HIV Infection in the Cross-Sectional Sample}

Table 2 presents age-adjusted odds ratios (aOR) for putative risk factors in the cross-sectional sample for prevalent HIV infection (by HIV rapid testing and ELISA), and recent infection (by STARHS assays, alone or in combination), compared to HIV-negative participants. Only number of sex partners in the prior 3 months (60-75 versus 76-120, aOR 5.1, $95 \%$ CI $(1.0,25.1)$ ) was associated with RI (by BED/Ax-AI combined), but not prevalent HIV infection. Two factorsHSV-2 co-infection and having recently sought treatment for a sexually transmitted infection (STI) - were positively associated with both prevalent infection (aOR $8.4(4.8,14.7)$ and $2.1(1.3$, 3.3), respectively) and RI by BED/Ax-AI combined (aOR 17.9 $(2.4,134.7)$, and $3.9(1.4,10.4)$, respectively). Several factors were associated (either positively or negatively) with prevalent $\mathrm{HIV}$, but not RI (by BED/Ax-AI combined), including: history of forced sex (aOR $2.2(1.5,3.1)$ ); current breastfeeding (aOR $0.3(0.2,0.4)$ ); being widowed (aOR $1.7(1.0,2.8)$ ); lifetime HIV testing history ( $\geq 2$ tests versus none, aOR $0.3(0.2,0.5) ; 1$ test versus none, aOR $0.6(0.4,0.9))$; regular alcohol consumption (aOR $1.5(1.1,2.2)$ ); and history of imprisonment (aOR $1.8(1.3,2.6))$. Education level, number of lifetime pregnancies, condom use, frequency of vaginal sex, and marital status were not significantly associated with prevalent or recent HIV infection.

Table 2 also presents risk factors for recent HIV infection on the BED and Ax-AI assays analyzed separately. HSV-2 seropositivity and recent STI treatment were consistently identified as risk factors in both models. However, the AxAI method identified two additional factors - district of residence and HIV testing history ( $\geq 2$ times versus never) that were not significant in the BED model. Risk factor associations for LTI, which excludes recent infections, and prevalent HIV, which includes RI, were nearly identical to one another (data not shown).

\section{Comparison of Risk Factors Associated with STARHS Results to those Identified via Prospectively Observed Seroconversion}

In the prospective cohort, having been HIV tested $\geq 2$ times versus never in one's lifetime and HIV testing within the past 6 months were positively associated with HIV seroconversion (Table 2). Having had two versus $\geq 4$ lifetime pregnancies was borderline statistically significant (aOR 8.0 $(1.0,65.4), P=0.05)$. None of the risk factors for prospective seroconversion was also identified as a risk factor for RI (by $\mathrm{BED} / \mathrm{Ax}-\mathrm{AI}$ combined) in the cross-sectional sample.

\section{DISCUSSION}

In these samples of urban Rwandan FSW, we found mixed agreement between three different methods of 
Table 1. Demographic and Behavioral Characteristics ${ }^{\mathrm{a}}$ of Female Sex Workers in Kigali, Rwanda

\begin{tabular}{|c|c|c|c|}
\hline $\begin{array}{l}\text { Median age, in years }(\mathrm{IQR})^{* * *} \\
\text { Age groups: * } \\
18-24 \\
25-29 \\
30-34 \\
\geq 35\end{array}$ & $\begin{array}{l}27.0(23-32) \\
66(34) \\
52(27) \\
41(21) \\
33(17)\end{array}$ & $\begin{array}{l}25.0(23-31) \\
86(41) \\
63(30) \\
29(14) \\
33(16)\end{array}$ & $\begin{array}{l}24.0(22-28) \\
199(50) \\
116(29) \\
48(12) \\
32(8)\end{array}$ \\
\hline $\begin{array}{l}\text { Education level: * } \\
\text { No formal schooling } \\
\text { Some primary school } \\
\text { Completed primary school } \\
\text { Secondary school (partial or completed) }\end{array}$ & $\begin{array}{l}45(23) \\
77(40) \\
48(25) \\
22(12) \\
\end{array}$ & $\begin{array}{l}35(17) \\
97(46) \\
46(22) \\
31(15) \\
\end{array}$ & $\begin{array}{l}87(22) \\
158(40) \\
111(28) \\
41(10)\end{array}$ \\
\hline $\begin{array}{l}\text { Lifetime no. pregnancies } \\
\qquad \begin{array}{l}\text { None } \\
1 \\
2 \\
3 \\
\geq 4\end{array}\end{array}$ & $\begin{array}{l}14(7) \\
52(27) \\
52(27) \\
34(18) \\
39(20)\end{array}$ & $\begin{array}{l}14(7) \\
60(29) \\
57(27) \\
36(17) \\
43(21)\end{array}$ & $\begin{array}{c}28(7) \\
119(30) \\
113(29) \\
77(19) \\
60(15)\end{array}$ \\
\hline Currently breastfeeding* & $40(21)$ & $95(45)$ & $219(55)$ \\
\hline $\begin{array}{l}\text { Marital status }{ }^{\mathrm{c}} \text { : } \\
\text { Married (legal or common-law marriage) } \\
\text { Divorced/separated* } \\
\text { Widowed } \\
\text { Never married* } \\
\text { Currently have steady partner }{ }^{\mathrm{d} * * *}\end{array}$ & $\begin{array}{c}0 \\
24(13) \\
37(19) \\
130(68) \\
62(32)\end{array}$ & $\begin{array}{c}0 \\
40(19) \\
22(10) \\
148(70) \\
92(44)\end{array}$ & $\begin{array}{c}4(1) \\
55(14) \\
34(9) \\
303(76) \\
122(31)\end{array}$ \\
\hline $\begin{array}{l}\text { No. vaginal sex acts in last month } \\
\quad<20 \\
20-39 \\
40-59 \\
60-89 \\
\geq 90\end{array}$ & $\begin{array}{l}31(16) \\
57(30) \\
42(22) \\
45(23) \\
17(9)\end{array}$ & $\begin{array}{l}32(15) \\
52(25) \\
54(26) \\
52(25) \\
21(10)\end{array}$ & $\begin{array}{c}73(18) \\
120(30) \\
81(20) \\
78(20) \\
45(11)\end{array}$ \\
\hline $\begin{array}{l}\text { No. sex partners in last } 3 \text { months } \\
\begin{array}{l}3-30 \\
31-59 \\
60-75 \\
76-120 \\
\geq 121\end{array}\end{array}$ & $\begin{array}{l}22(12) \\
25(13) \\
37(19) \\
34(18) \\
74(39)\end{array}$ & $\begin{array}{c}8(4) \\
28(13) \\
25(12) \\
48(23) \\
102(48)\end{array}$ & $\begin{array}{c}41(10) \\
68(17) \\
59(15) \\
81(21) \\
146(37)\end{array}$ \\
\hline $\begin{array}{l}\text { No. clients per week in last month } \\
\quad<5 \\
5-9 \\
10-15 \\
16-25 \\
>25\end{array}$ & $\begin{array}{l}32(17) \\
59(31) \\
52(27) \\
39(20) \\
10(5)\end{array}$ & $\begin{array}{l}31(15) \\
53(25) \\
66(31) \\
42(20) \\
19(9)\end{array}$ & $\begin{array}{c}87(22) \\
104(26) \\
110(28) \\
66(17) \\
30(8)\end{array}$ \\
\hline
\end{tabular}


(Table 1) contd.....

\begin{tabular}{|c|c|c|c|}
\hline & $\begin{array}{c}\text { HIV-Positive Cross-Sectional } \\
\text { Survey Participants } \\
(\mathrm{N}=192) \\
\text { N }(\%)\end{array}$ & $\begin{array}{c}\text { HIV-Negative, Non-Enrolled } \\
\text { Survey Participants } \\
(\mathbf{N}=211) \\
\text { N }(\%)\end{array}$ & $\begin{array}{c}\text { Prospective Cohort Participants } \\
(\mathbf{N}=397) \\
\text { N }(\%)\end{array}$ \\
\hline \multicolumn{4}{|l|}{ No. years working as sex worker* } \\
\hline$\leq 1$ & $43(22)$ & $45(21)$ & $89(22)$ \\
\hline $4-5$ & $35(18)$ & $48(23)$ & $70(18)$ \\
\hline$\geq 6$ & $54(28)$ & $52(25)$ & $75(19)$ \\
\hline Ever had forced sex** & $72(38)$ & $55(26)$ & $76(19)$ \\
\hline \multicolumn{4}{|l|}{ No. lifetime HIV tests (IQR) } \\
\hline Never tested & $97(51)$ & $72(34)$ & $119(30)$ \\
\hline$\geq 6$ times & $2(1)$ & 0 & $13(3)$ \\
\hline HIV testing in the last 6 months & $4(2)$ & $22(11)$ & $57(15)$ \\
\hline$\geq 1$ Genital symptom in past month & $50(26)$ & $50(24)$ & $78(20)$ \\
\hline Sought treatment for STI symptom in last 3 months & $34(18)$ & $22(10)$ & $32(8)$ \\
\hline Drink alcohol regularly & $120(63)$ & $119(56)$ & $203(51)$ \\
\hline Have sex with clients who have consumed alcohol & $185(97)$ & $196(93)$ & $371(94)$ \\
\hline Ever imprisoned & $89(47)$ & 77 (37) & $150(38)$ \\
\hline 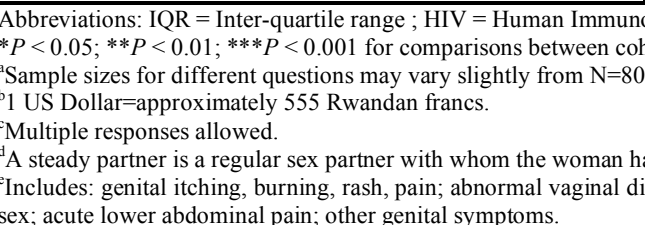 & $\begin{array}{l}\text { deficiency Virus; STI }=\text { Sexually tra } \\
\text { ort participants and HIV-negative, no } \\
0 \text { based on missing responses. } \\
\text { as sex more often than with other part } \\
\text { scharge, odor, or bleeding (non-mens }\end{array}$ & $\begin{array}{l}\text { nsmitted infections. } \\
\text { n-enrolled survey participants. }\end{array}$ & $\begin{array}{l}\text { ot married to. } \\
\text { nital ulcers, sores or blisters; pain during }\end{array}$ \\
\hline
\end{tabular}

assessing risk factors for HIV infection, even when the HIV incidence rates based on $\mathrm{BED}$ alone, $\mathrm{BED} / \mathrm{Ax}-\mathrm{AI}$ combined, and observed seroconversion were relatively similar [24]. We identified a number of putative and protective risk factors for prevalent HIV infection using conventional serologic testing. We identified only a few risk factors for recent infection using two STARHS assays (BED and AxAI) with three different classifications. Although two of the three factors (HSV-2 coinfection and recent STI treatment) were also risk factors for prevalent infection, the third factor (number of sex partners in the past 3 months) was only identified by the STARHS classification that best classified recent HIV infection in this sample [24], the BED/Ax-AI combined. Finally, risk factors for recent infection (by single or combined STARHS assays) were different from those associated with HIV seroconversions in the prospective cohort.

Decisions by ministries of health to institutionalize the use of STARHS for incidence surveillance-specifically, to generate HIV incidence estimates and identify risk factors for recently acquired infection from cross-sectional samples in settings such as Kigali, Rwanda-hinge on the epidemiologic utility of the approach. We defined epidemiologic utility, in part, as the ability of an assay or algorithm to distinguish risk factors for recent from longerterm infection when such differences exist. From the perspective of epidemiologic utility, factors associated with prevalent infection differed substantially from those associated with recent infection in this sample.

Our findings are consistent with at least two plausible scenarios in this specific population of Rwandan female sex workers. First, in reality, there may be differences between factors associated with incident versus prevalent HIV infection in this population, for example if HIV transmission dynamics in this group have recently begun to evolve. Alternatively, true similarities in the risk factor profile for incident and prevalent infection in this population could have been masked in our analyses by misclassification on the assays or low statistical power to identify risk factors as statistically significant. However, prevalent infections, recent infections by STARHS, and prospectively observed seroconversions measure varying stages of infection in different time periods, and so risk factors could be expected to be different when the epidemic is evolving. Nonetheless, the use of epidemiologic utility, including assay sensitivity and specificity, as a metric of STARHS is conceptually and methodologically important, particularly given the intent to use STARHS as an epidemiological tool at the population level, not at the individual level [38]. Future studies, ideally with more statistical power, are needed to further examine the epidemiologic utility among at-risk sub-Saharan African populations. 
Table 2. Risk Factors for Prevalent HIV Infection, Recent HIV Infection ${ }^{\text {a }}$ (by BED-CEIA Assay and Ax-AI, Separately and Combined) and HIV Seroconversion in a Sample of ART-Naïve, Rwandan Female Sex Workers

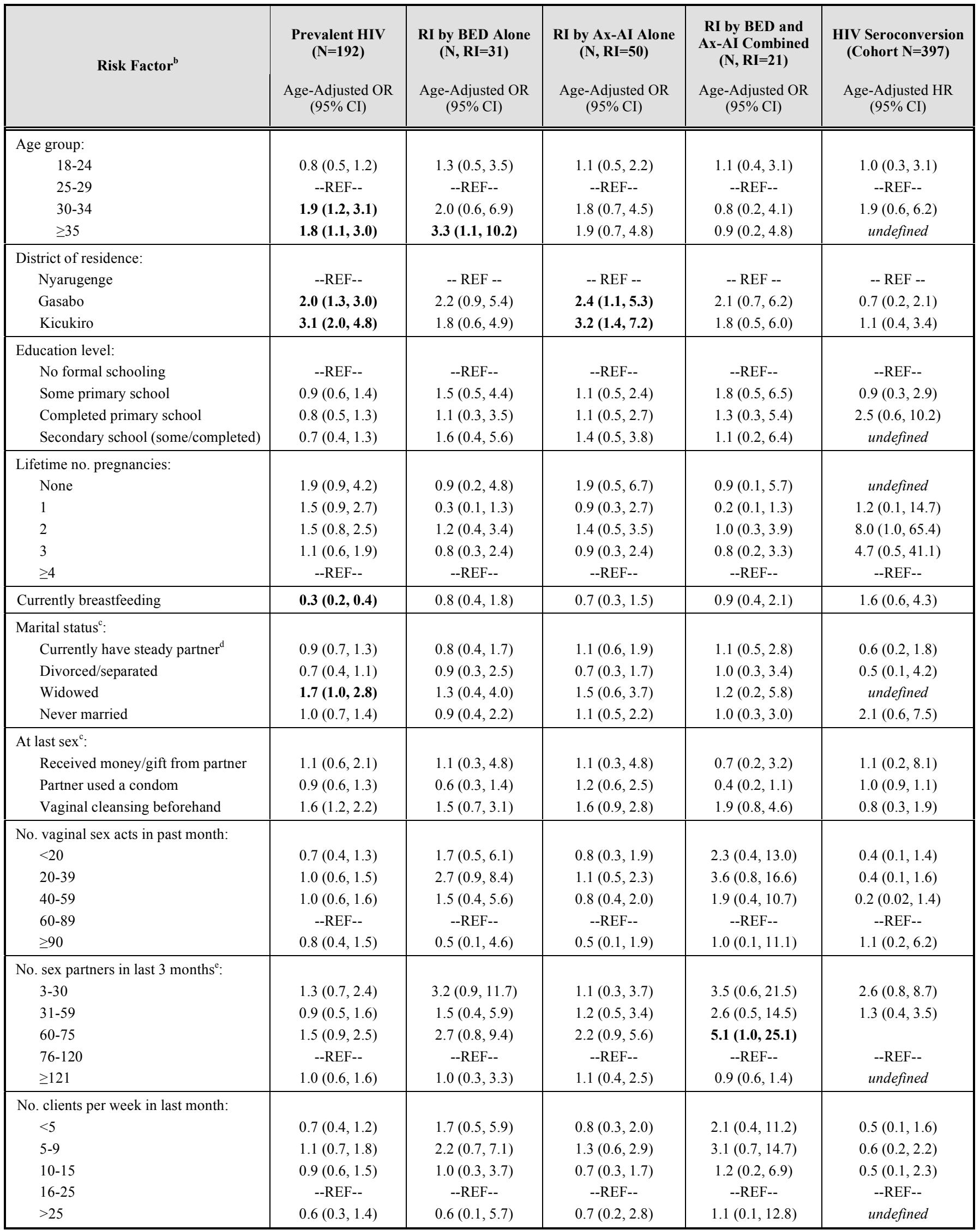


(Table 2) contd.....

\begin{tabular}{|c|c|c|c|c|c|}
\hline Risk Factor $^{\text {b }}$ & $\begin{array}{c}\begin{array}{c}\text { Prevalent HIV } \\
(\mathbf{N}=192)\end{array} \\
\begin{array}{c}\text { Age-Adjusted OR } \\
(95 \% \mathrm{CI})\end{array}\end{array}$ & $\begin{array}{l}\text { RI by BED Alone } \\
\text { (N, RI=31) } \\
\text { Age-Adjusted OR } \\
\quad(95 \% \mathrm{CI})\end{array}$ & $\begin{array}{l}\text { RI by Ax-AI Alone } \\
\quad(\mathbf{N}, \mathbf{R I}=\mathbf{5 0}) \\
\text { Age-Adjusted OR } \\
(95 \% \mathrm{CI})\end{array}$ & $\begin{array}{l}\text { RI by BED and } \\
\text { Ax-AI Combined } \\
\text { (N, RI=21) } \\
\text { Age-Adjusted OR } \\
\quad(95 \% \mathrm{CI})\end{array}$ & $\begin{array}{l}\text { HIV Seroconversion } \\
\text { (Cohort N=397) } \\
\text { Age-Adjusted HR } \\
(95 \% \mathrm{CI})\end{array}$ \\
\hline $\begin{array}{l}\text { No. years working as sex worker }{ }^{\mathrm{f}} \text { : } \\
\quad \leq 1 \\
2 \\
3 \\
4-5 \\
\geq 6\end{array}$ & $\begin{array}{c}1.0(0.6,1.7) \\
\mathbf{0 . 4}(\mathbf{0 . 2}, \mathbf{0 . 8}) \\
1.2(0.7,1.9) \\
0.8(0.5,1.4) \\
-- \text { REF-- }\end{array}$ & $\begin{array}{c}1.9(0.7,5.6) \\
0.8(0.2,2.8) \\
0.6(0.1,2.3) \\
0.8(0.3,2.6) \\
-- \text { REF-- }\end{array}$ & $\begin{array}{c}1.5(0.7,3.6) \\
0.3(0.1,1.1) \\
1.0(0.4,2.6) \\
0.7(0.2,1.7) \\
-- \text { REF-- }\end{array}$ & $\begin{array}{c}4.2(0.9,19.4) \\
1.0(0.1,6.9) \\
1.9(0.3,10.7) \\
1.4(0.2,8.8) \\
\text {--REF-- }\end{array}$ & $\begin{array}{l}\text {--REF-- } \\
\text { undefined }\end{array}$ \\
\hline Ever had forced sex & $2.2(1.5,3.1)$ & $1.7(0.8,3.7)$ & $1.8(1.0,3.4)$ & $2.3(0.9,5.7)$ & $0.8(0.2,2.6)$ \\
\hline $\begin{array}{l}\text { Lifetime no. HIV tests } \\
\text { None } \\
\quad 1 \\
\quad \geq 2\end{array}$ & $\begin{array}{c}--\mathrm{REF}-- \\
0.6(0.4,0.9) \\
0.3(0.2,0.5)\end{array}$ & $\begin{array}{c}\text {--REF-- } \\
0.6(0.3,1.5) \\
0.6(0.3,1.5)\end{array}$ & $\begin{array}{c}\text {--REF-- } \\
0.6(0.3,1.2) \\
\mathbf{0 . 4}(\mathbf{0 . 2}, \mathbf{0 . 9})\end{array}$ & $\begin{array}{c}\text {--REF-- } \\
0.6(0.2,1.9) \\
1.1(0.4,3.0)\end{array}$ & $\begin{array}{c}\text {--REF-- } \\
4.8(0.6,41.2) \\
\mathbf{1 2 . 9}(\mathbf{1 . 7 , 9 8 . 9 )}\end{array}$ \\
\hline HIV tested in last 6 months & $0.2(0.1,0.4)$ & $0.2(0.1,1.8)$ & $0.1(0.01,1.0)$ & $0.3(0.04,2.2)$ & $3.4(1.2,9.1)$ \\
\hline Sought STI treatment in last 3 months & $2.1(1.3,3.3)$ & $2.8(1.2,6.9)$ & $3.1(1.5,6.2)$ & $3.9(1.4,10.4)$ & $0.6(0.1,4.3)$ \\
\hline Drink alcohol regularly & $1.5(1.1,2.2)$ & $1.1(0.5,2.3)$ & $1.3(0.7,2.3)$ & $0.7(0.3,1.7)$ & $1.3(0.5,3.3)$ \\
\hline Ever imprisoned & $1.8(1.3,2.6)$ & $0.9(0.4,2.0)$ & $1.2(0.6,2.2)$ & $0.6(0.2,1.7)$ & $1.4(0.5,3.5)$ \\
\hline HSV-2 seropositive (baseline) & $8.4(4.8,14.7)$ & $3.9(1.5,10.5)$ & $9.1(3.2,25.9)$ & $17.9(2.4,134.7)$ & $1.4(0.5,3.8)$ \\
\hline Pregnant (baseline) & $0.7(0.3,1.3)$ & $0.8(0.2,3.5)$ & $0.7(0.2,2.5)$ & $1.1(0.3,5.0)$ & NA \\
\hline $\begin{array}{l}\text { Abbreviations: } \mathrm{OR}=\text { odds ratio; } \mathrm{HR}=\text { hazard rat } \\
\text { pplicable. } \\
\text { 3olded effect estimates have } P<0.05 \text {. } \\
\text { Participants with CD4<200 removed from RI } \\
\text { Sample sizes for different questions may vary } \\
\text { Multiple responses allowed. } \\
\text { A steady partner is a regular sex partner with } \\
\text { Categories for age-adjusted HR's for prospec } \\
\text { Categories for age-adjusted HR's for prospect }\end{array}$ & $\begin{array}{l}\text { ication, considered } \\
\text { ly from the total } \mathrm{N} \mathrm{b} \\
\text { the woman has sex } \\
\text { hort are: } 3-30 ; 31-7 \\
\text { hort are: } \leq 1 ; 2-3 \text {; an }\end{array}$ & $\begin{array}{l}\text { le long-term HIV in } \\
\text { on missing responses } \\
\text { often than with other } \\
\geq 76 \text { partners (refere } \\
\text { ears (referent group }\end{array}$ & $\begin{array}{l}\text { artner(s), but does } n \\
\text { up is } \geq 76) \text {. }\end{array}$ & evith & to. \\
\hline
\end{tabular}

We conducted a number of sensitivity analyses of our data. Specifically, adjustment of STARHS results from bivariable models with $\mathrm{CD} 4$ count data (with individuals with $\mathrm{CD} 4<200$ cells $/ \mu$ l excluded as probable LTI) did not change inference regarding the existence of associations for specific risk factors. Additional sensitivity analyses (data not shown) showed that excluding persons classified as RI with CD4 counts up to 500 cells $/ \mu 1$ did not alter risk factor findings. Misclassification rates may change with different study populations, for example a less healthy population with a different CD4 count distribution; misclassification of individuals with LTI and high CD4 counts is also possible. In our study, it is possible that greater specificity in the risk factor analysis from use of the BED and Ax-AI assays combined reduced the potential impact of CD4 adjustment. Indeed, the combined testing algorithm appeared to perform better for risk factor identification in the cross-sectional sample than the assays alone, particularly compared with the Ax-AI method alone. For example, the two additional risk factors for RI identified by the Ax-AI method alone were identified as risk factors for prevalent infection, which reflects the substantial misclassification of individuals with longer-term infection as RI by the Ax-AI method in this population [24]. CD4 adjustment may have a greater impact in studies using only a single assay versus a combined algorithm, and such studies should therefore conduct CD4 testing if possible. Finally, conducting STARHS studies among treatment-naïve individuals will help avoid misinterpretation of STARHS results with CD4 adjustment.

Comparison of the risk factors identified using the crosssectional methods against those identified using the goldstandard prospective method may aid the interpretation of cross-sectional risk factor data, however caution when making such a comparison is warranted. In this study, there was no overlap between risk factors for recent infection in the cross-sectional sample and predictors of HIV seroconversion in the prospective sub-sample. Limited statistical power for identifying significant factors could have affected agreement between the methods, as there were relatively few events in both the RI and seroconversion models. However, important differences between the subsample of women who were eligible and agreed to participate in the prospective cohort study and HIV-negative survey participants not enrolled in the cohort may also contribute to the discordance. For example, HIV-negative cohort participants were younger, more likely to be breastfeeding, less likely to have a history of forced sex, and had more recently initiated sex work than HIV-negative nonenrolled women, all factors associated with HIV risk. Furthermore, changes over time in cohort participants' risk level (for example, because of the effect of prevention interventions or the Hawthorne effect), or unknown factors 
Table 3. Comparison of Demographic and Risk Characteristics of HIV Seroconverters (N=19) and HIV-Negative Participants (N=378), Prospective Cohort, Kigali, Rwanda

\begin{tabular}{|c|c|c|}
\hline & $\begin{array}{l}\text { Seroconverters } \\
\text { (N=19) } \\
\mathbf{N}(\%)\end{array}$ & $\begin{array}{c}\text { HIV-Negative } \\
\text { Cohort Participants } \\
(\mathrm{N}=378) \\
\text { N }(\%)\end{array}$ \\
\hline Median age (IQR) & $25.0(22-31)$ & $24.0(22-28)$ \\
\hline \multicolumn{3}{|l|}{ Age group, years: } \\
\hline $18-24$ & $8(42)$ & $191(51)$ \\
\hline $25-29$ & $5(26)$ & $111(30)$ \\
\hline$\geq 30$ & $6(32)$ & $74(20)$ \\
\hline \multicolumn{3}{|l|}{ Education level: } \\
\hline No school & $4(21)$ & $83(22)$ \\
\hline Primary (some/completed) & $11(58)$ & $258(68)$ \\
\hline Some secondary & $4(21)$ & $37(10)$ \\
\hline Currently breastfeeding & $12(63)$ & $207(55)$ \\
\hline \multicolumn{3}{|l|}{ Lifetime no. pregnancies: } \\
\hline None or 1 & $2(11)$ & $145(38)$ \\
\hline 2 & $11(58)$ & $102(27)$ \\
\hline 3 & $5(26)$ & $72(19)$ \\
\hline$\geq 4$ & $1(5)$ & $59(16)$ \\
\hline Male condom use at last sex & $16(84)$ & $280(74)$ \\
\hline Current hormonal contraception use & $5(26)$ & $67(18)$ \\
\hline$\geq 1$ Genital symptom in past month ${ }^{+}$ & $6(32)$ & $72(19)$ \\
\hline \multicolumn{3}{|l|}{ No. vaginal sex acts in past month: } \\
\hline$<20$ & $6(32)$ & $67(18)$ \\
\hline $20-39$ & $4(21)$ & $116(31)$ \\
\hline $40-59$ & $1(5)$ & $80(21)$ \\
\hline $60-89$ & $5(26)$ & $73(19)$ \\
\hline$\geq 90$ & $3(16)$ & $42(11)$ \\
\hline \multicolumn{3}{|l|}{ No. sex partners in last 3 months: } \\
\hline $3-30$ & $4(21)$ & $37(10)$ \\
\hline $31-75$ & $6(32)$ & $121(32)$ \\
\hline$\geq 76$ & $9(47)$ & $218(58)$ \\
\hline \multicolumn{3}{|l|}{ No. clients per week in past month: } \\
\hline$<5$ & $6(32)$ & $81(21)$ \\
\hline $5-9$ & $4(21)$ & $100(27)$ \\
\hline $10-15$ & $2(11)$ & $108(29)$ \\
\hline$\geq 16$ & $7(36)$ & $89(23)$ \\
\hline \multicolumn{3}{|l|}{ No. years working as sex worker ${ }^{\ddagger}$ : } \\
\hline$\leq 1$ & $6(35)$ & $61(17)$ \\
\hline $2-3$ & $3(18)$ & $160(45)$ \\
\hline$\geq 4$ & $8(47)$ & $137(38)$ \\
\hline \multicolumn{3}{|l|}{ No. lifetime HIV tests: } \\
\hline Never tested & $1(5)$ & $118(31)$ \\
\hline Once & $5(26)$ & $132(35)$ \\
\hline$\geq$ Twice & $13(69)$ & $127(34)$ \\
\hline HIV tested in prior 6 months & $6(32)$ & $51(14)$ \\
\hline HSV-2 seropositive at baseline & $12(67)$ & $202(56)$ \\
\hline Median days from enrollment to seroconversion (range) & $269(86-768)$ & NA \\
\hline
\end{tabular}

Abbreviations: IQR = Inter-quartile range; HIV = Human Immunodeficiency Virus; NA = Not applicable.

${ }^{+}$Includes: genital itching, burning, rash, pain; abnormal vaginal discharge, odor, or bleeding (non-menstrual); pain or difficulty urinating; genital ulcers, sores or blisters; pain during sex; acute lower abdominal pain; other genital symptoms.

${ }^{\ddagger} 2$ participants missing response, $\mathrm{N}=17$. 
causing misclassification on the assays, could also have contributed to discordance between cross-sectional and prospective risk factor findings. Most HIV seroconversions in the cohort occurred early during follow-up, and women who seroconverted differed from women who remained HIV-negative during follow-up on several potentially important factors. For example, seroconverters had spent fewer years in sex work, reported more frequent sex, had higher HSV-2 seroprevalence at baseline, and reported more genital symptoms in the month prior to enrollment (Table 3 ). Additional study limitations are noted. Due to sample size (specifically, too few outcomes), we could only adjust for age and thus were unable to conduct a full multivariate analysis. This could have resulted in uncontrolled confounding, complicating comparisons between prospective and cross-sectional samples. Measurement error in covariates and bias due to unmeasured confounding could have affected risk factor findings.

This study also has several strengths and provides new insights. The use of two STARHS assays contributed important information about the assays' combined and individual performance in a high-prevalence population. The combined cross-sectional and prospective design, and extensive interview data, enabled in-depth exploration, triangulation, and comparison of risk factors for prevalent and recently acquired HIV infection in multiple samples. This in turn allowed us to explore an important dimension of the epidemiologic utility of HIV incidence assays, namely risk factor detection. Finally, availability of $\mathrm{CD} 4$ count data aided interpretation and enabled important sensitivity analyses.

\section{CONCLUSION}

In this selected high-risk group, factors associated with incident HIV infection appeared to differ from those associated with prevalent infection. More studies evaluating the ability of STARHS to detect risk factors when combined with survey data, alongside the validity of STARHS-based incidence estimates, are needed to further establish the epidemiologic utility of the approach for incidence surveillance. Such studies are especially needed in subSaharan Africa where there is very limited experience with STARHS, and where the potential impact of an alternative to prospective measurement of HIV incidence is greatest [5]. Specifically, such investigations should be conducted in populations in which risk factors for HIV incidence and prevalence are known or expected to differ, as well as populations with more diversity in risk factors (e.g., population-based samples). Despite persisting concerns about the validity of particular STARHS assays, the results of this study underscore the potential value of the approach in identifying and distinguishing groups in need of HIV prevention services when combined with complementary risk group and behavioral information.

Public health officials in Rwanda might consider incorporating STARHS into the next Demographic and Health Survey-Plus to conduct similar analyses on the national level and across population groups, as has been done successfully in Uganda [37] and South Africa [18]. Further evaluations of STARHS data in Rwanda and across diverse settings, as well as development of assays with improved validity, will strengthen the argument to incorporate HIV incidence surveillance into routine HIV surveillance.

\section{ACKNOWLEDGEMENTS}

The authors acknowledge the following groups and individuals for their contributions to this study: Projet Ubuzima study team; Projet Ubuzima Governing Council, specifically Dr. Anita Assimwe; Projet Ubuzima Community Advisory Group and community mobilizers; staff at the National Reference Laboratory in Kigali, including John Rusine and Odette Mukabayire; staff at the Academic Medical Center-Center for Poverty-related and Communicable Diseases, specifically Robert Meester; clinical affairs team at the International Partnership for Microbicides, Inc.; Dr. Paul Coplan, Dr. Eugénie Kayirangwa, and Dr. Willi McFarland for their contributions to the design and planning of the study.

This work was supported by the International Partnership for Microbicides, Inc., the European and Developing Countries Clinical Trials Partnership (through a project entitled: "Preparing for Phase III vaginal microbicide trials in Rwanda and Kenya: Preparedness studies, capacity building, and strengthening of medical referral systems"), the United States Centers for Disease Control and Prevention, and National Institutes of Health - Institutional Training Grant. The funders had no role in study design, data collection and analysis, decision to publish, or preparation of the manuscript.

The findings and conclusions in this report are those of the authors and do not necessarily represent the official position of the US Centers for Disease Control and Prevention or the European and Developing Countries Clinical Trials Partnership.

\section{CONFLICT OF INTEREST}

The authors confirm that this article content has no conflicts of interest.

\section{REFERENCES}

[1] UNAIDS. Trends in HIV incidence and prevalence: natural course of the epidemic or results of behavioral change? UNAIDS Best practices collection. 1999; pp. 1-32.

[2] Suligoi B, Wagner TM, Ciccozzi M, Rezza G. The epidemiological contribution to the preparation of field trials for HIV and STI vaccines: objectives and methods of feasibility studies. Vaccine 2005; 23(12): 1437-45.

[3] Diaz T, De Cock K, Brown T, Ghys PD, Boerma JT. New strategies for HIV surveillance in resource-constrained settings: an overview. AIDS (London, England) 2005; 19(Suppl 2): S1-8.

[4] UNAIDS. 2008 Report on the Global AIDS Epidemic. Geneva: UNAIDS. August 2008.

[5] Braunstein SL, van de Wijgert JH, Nash D. HIV incidence in subSaharan Africa: a review of available data with implications for surveillance and prevention planning. AIDS Rev 2009; 11(3): 14056.

[6] MEASURE. Demographic and Health Surveys. Available from: http://www.measuredhs.com/aboutsurveys/dhs/start.cfm [Cited 2011 January 17].

[7] CDC. Guidelines for National Human Immunodeficiency Virus Case Surveillance, including monitoring for human immunodeficiency virus infection and acquired immunodeficiency syndrome. MMRW 1999; 48(RR13): 1-28

[8] Lee LM, McKenna MT. Monitoring the Incidence of HIV Infection in the United States. Public Health Rep 2007; 122(Suppl 1): 72-9. 
[9] Hall HI, Song R, Rhodes P, et al. Estimation of HIV incidence in the United States. JAMA 2008; 300(5): 520-9.

[10] Workshop on the Serological Testing Algorithm for Recent HIV Seroconversion (STARHS) and HIV incidence estimates, Stockholm, 11-12 March 2008. Euro Surveill 2008; 13(36): 1-2.

[11] World Health Organization. Priority Interventions: HIV/AIDS prevention, treatment, and care in the health sector. Geneva: World Health Organization September 2008.

[12] Parekh BS, Kennedy MS, Dobbs T, et al. Quantitative detection of increasing HIV type 1 antibodies after seroconversion: a simple assay for detecting recent HIV infection and estimating incidence. AIDS Res Hum Retroviruses 2002; 18(4): 295-307.

[13] Suligoi B, Massi M, Galli C, et al. Identifying recent HIV infections using the avidity index and an automated enzyme immunoassay. J Acquir Immune Defic Syndr 2003; 32(4): 424-8.

[14] Hu DJ, Vanichseni S, Mock PA, et al. HIV type 1 incidence estimates by detection of recent infection from a cross-sectional sampling of injection drug users in Bangkok: use of the $\operatorname{IgG}$ capture BED enzyme immunoassay. AIDS Res Hum Retroviruses 2003; 19(9): 727-30.

[15] Jiang Y, Wang M, Ni M, et al. HIV-1 incidence estimates using IgG-capture BED-enzyme immunoassay from surveillance sites of injection drug users in three cities of China. AIDS (London, England) 2007; 21(Suppl 8): S47-51.

[16] Wolday D, Meles H, Hailu E, et al. Temporal trends in the incidence of HIV infection in antenatal clinic attendees in Addis Ababa, Ethiopia, 1995-2003. J Intern Med 2007; 261(2): 132-7.

[17] Sakarovitch C, Rouet F, Murphy G, et al. Do tests devised to detect recent HIV-1 infection provide reliable estimates of incidence in Africa? J Acquir Immune Defic Syndr 2007; 45(1): 115-22.

[18] Rehle T, Shisana O, Pillay V, Zuma K, Puren A, Parker W. National HIV incidence measures--new insights into the South African epidemic. S Afr Med J 2007; 97(3): 194-9.

[19] Karita E, Price M, Hunter E, et al. Investigating the utility of the HIV-1 BED capture enzyme immunoassay using cross-sectional and longitudinal seroconverter specimens from Africa. AIDS (London, England) 2007; 21(4): 403-8.

[20] Hargrove JW, Humphrey JH, Mutasa K, et al. Improved HIV-1 incidence estimates using the BED capture enzyme immunoassay. AIDS (London, England) 2008; 22(4): 511-8.

[21] Bulterys M, Parekh BS, Chao A, et al. Use of an IgG capture BED enzyme immunoassay to investigate recent HIV-1 seroconversions among young women in Rwanda: Comparison with 24-month prospective cohort data. International AIDS Conference July 2004; Bangkok, Thailand.

[22] Barnighausen T, Tanser F, Gqwede Z, Mbizana C, Herbst K, Newell ML. High HIV incidence in a community with high HIV prevalence in rural South Africa: findings from a prospective population-based study. AIDS (London, England) 2008; 22(1): 139-44.

[23] Kim AA, Hallett T, Stover J, et al. Estimating HIV incidence among adults in Kenya and Uganda: a systematic comparison of multiple methods. PLoS One 2011; 6(3): e17535.
[24] Braunstein S, Nash D, Kim A, et al. Dual testing algorithm of BED-CEIA and AxSYM Avidity Index assays performs best in identifying recent HIV infection in a sample of Rwandan sex workers. PLoS One 2011 Ap; 6(4): e18402.

[25] Ghys PD, Kufa E, George MV. Measuring trends in prevalence and incidence of HIV infection in countries with generalised epidemics. Sex Transm Infect 2006; 82(Suppl 1): i52-6.

[26] Garnett GP, Gregson S, Stanecki KA. Criteria for detecting and understanding changes in the risk of HIV infection at a national level in generalised epidemics. Sex Transm Infect 2006; 82(Suppl 1): i48-51.

[27] Marinda ET, Hargrove J, Preiser W, et al. Significantly diminished long-term specificity of the BED Capture Enzyme Immunoassay among patients with HIV-1 with very low CD4 counts and those on antiretroviral therapy. J Acquir Immune Defic Syndr 2010; 53(4): 496-500.

[28] Hallett T, Garnett G. Estimating incidence of HIV infection in Uganda. JAMA 2009; 301(2): 159; author reply 60-1.

[29] Todd J, Lutalo T, Kaleebu P. Estimating incidence of HIV infection in Uganda. JAMA 2009; 301(2): 159-60; author reply 601.

[30] Mermin J, Musinguzi J, Hladik W. Estimating incidence of HIV infection in Uganda - Reply. JAMA 2009; 301(2): 160-1.

[31] Parekh BS, McDougal JS. Application of laboratory methods for estimation of HIV-1 incidence. Indian J Med Res 2005; 121(4): 510-8.

[32] Chawla A, Murphy G, Donnelly C, et al. Human immunodeficiency virus (HIV) antibody avidity testing to identify recent infection in newly diagnosed HIV type 1 (HIV-1)-seropositive persons infected with diverse HIV-1 subtypes. J Clin Microbiol 2007; 45(2): 415-20.

[33] Hayashida T, Gatanaga H, Tanuma J, Oka S. Effects of Low HIV Type 1 Load and Antiretroviral Treatment on IgG-Capture BEDEnzyme Immunoassay. AIDS Res Hum Retroviruses 2008; 24(3): 495-8.

[34] Hallett TB, Ghys P, Barnighausen T, Yan P, Garnett GP. Errors in 'BED'-derived estimates of HIV incidence will vary by place, time and age. PLoS One 2009; 4(5): e5720.

[35] Laeyendecker O, Brookmeyer R, Oliver AE, et al. for the MultiCenter AIDS Cohort Study (MACS). Factors associated with incorrect identification of recent HIV infection using the BED capture immunoassay. AIDS Res Hum Retroviruses 2011; [Epub ahead of print].

[36] Hayashida T, Gatanaga H, Tanuma J, Oka S. Effects of low HIV type 1 load and antiretroviral treatment on IgG-capture BEDenzyme immunoassay. AIDS Res Hum Retroviruses 2008; 24(3): 495-8.

[37] Mermin J, Musinguzi J, Opio A, et al. Risk factors for recent HIV infection in Uganda. JAMA 2008; 300(5): 540-9.

[38] Guy R, Gold J, Calleja JM, et al. Accuracy of serological assays for detection of recent infection with HIV and estimation of population incidence: a systematic review. Lancet Infect Dis 2009; 9(12): 74759. 